\title{
Slit Ventricle as a Neurosurgical Emergency: Case Report and Review of Literature
}

\section{Zoltan Mencser ${ }^{1}, Z^{2}$ solt Kopniczky ${ }^{1,2}$, David Kis ${ }^{1}$, Pal Barzo ${ }^{1}$}

\section{Key words \\ Hydrocephalus \\ - Raised ICP \\ - Shunt failure \\ - Slit ventricle}

\section{Abbreviations and Acronyms \\ CSF: Cerebrospinal fluid \\ ICP: Intracranial pressure}

From the ${ }^{\mathbf{1}}$ Department of Neurosurgery, Faculty of Medicine, Albert Szent-Györgyi Clinical Center, University of Szeged, Szeged; and ${ }^{2}$ Department of Neurotraumatology, Péterfy Hospital - National Institute of Traumatology, Budapest, Hungary

To whom correspondence should be addressed:

Zoltan Mencser, M.D.

[E-mail: mencser67@gmail.com]

Citation: World Neurosurg. (2019) 130:493-498.

https://doi.org/10.1016/j.wneu.2019.07.006

Journal homepage: www.journals.elsevier.com/worldneurosurgery

Available online: www.sciencedirect.com

1878-8750/\$ - see front matter (c) 2019 Elsevier Inc. All rights reserved.

\section{INTRODUCTION}

Symptomatic slit ventricle is one of the most challenging complications of shunt surgery in children. ${ }^{\mathrm{I}-4}$ The clinical triad features headache, slow refilling of the valve, and narrow ventricles on imaging. Most authors agree that the most likely underlying mechanism is chronic cerebrospinal fluid (CSF) overdrainage. However, clinical signs and symptoms may appear along with a wide range of intracranial pressure (ICP) values. Probably, as the Rekate classification suggests, ${ }^{4}$ multiple pathophysiologic mechanisms can be involved to produce these symptoms. The common features are previous hydrocephalus treated with shunt insertion and headache. ${ }^{4,5}$

The following 5 distinct syndromes were identified by Rekate:I) intermittent, extremely low-pressure headaches that are analogous to spinal headaches; 2) intermittent proximal obstruction; 3) shunt failure with small ventricles (normal volume hydrocephalus); 4) intracranial

BACKGROUND: Symptomatic slit ventricle is one of the most challenging complications of shunt surgery in children. Clinical signs and symptoms may appear with a wide range of intracranial pressure (ICP) values. We report the case of a 10-year-old girl, who did not present the classic clinical features of extremely elevated ICP, which was proven by multiple invasive ICP recordings, performed during shunt revisions.

CASE DESCRIPTION: At the age of 6 months, the patient presented squeal for many hours, accompanied with sunset eyes, bulging anterior fontanel, and dilated ventricles of all 4 ventricles on computed tomography scan. Acute ventriculoperitoneal shunt insertion was performed with adjustable valve. During the following 9 years, she was regularly seen and medically treated for intermittent headache, with nausea and vomiting. From 9 years of age, she was hospitalized for severe (10/10 on the visual analog scale), unbearable headache, agitation, and screaming on multiple occasions. Altogether, we had to revise the shunt system 5 times throughout 1 year. Radiologic imaging always showed narrow ventricles. Ophthalmologic examination of the fundus never revealed signs of raised ICP. Perioperative monitoring of the ICP with intraparenchymal sensor showed unexpected high values of $40-45 \mathrm{~mm} \mathrm{Hg}$. However, repetitive shunt revisions were successful only temporarily because the symptoms always returned. Only bilateral shunting of the ventricular system was able to eliminate the symptoms permanently.

CONCLUSIONS: Images of slit ventricle can be associated either with low or extremely high ICP needing urgent surgical consideration, including ICP monitoring. Bilateral shunt insertion can be effective treatment for slit ventricle syndrome.

hypertension with working shunts (hydrocephalic pseudotumor); and 5) headaches unrelated to shunt function.

The clinical appearance is marked with chronic or intermittent headache, associated nausea, vomiting, lethargy, and different degrees of consciousness or irritability.

Patients in the Rekate groups 2, 3, and 4 are at major risk when elevated ICP warrants urgent actions. ${ }^{7}$ Early recognition and adequate therapy are especially important in cases of groups 3 and 4 , which are characterized by intermittent shunt failure, causing increased ICP either in a form of spike waves or as a progressive elevation of baseline pressure. Urgent intervention is needed in a classic clinical form of raised ICP, such as confusion, drowsiness, slow psychomotor reactions, and vegetative reflexes (bradycardia and hypertension). ${ }^{4,8}$

\section{CASE PRESENTATION}

At 6 months of age, the patient presented squeal for several hours accompanied with sunset eyes sign and bulging anterior fontanel. The cranial ultrasound and head computed tomography scan showed dilated ventricles under tension of all 4 ventricles with left-sided porencephaly. She was diagnosed with hydrocephalus, and prompt insertion of a ventriculoperitoneal shunt system was performed with a programmable valve (Sophysa SM8 
[Sophysa, Orsay, France]). Acute clinical signs and symptoms improved as raised ICP returned to a normal level.

During the following 9 years, she was occasionally seen at our department for intermittent headache, which sometimes was associated with an upper respiratory tract infection and could be managed medically. She was admitted at the age of 4 years, when headache set on suddenly, and she was screaming because of intolerable pain. The pain disappeared after a deep sleep, but later reappeared again. However, resetting the valve opening pressure at a higher level and enhancing fluid intake relieved her symptoms without need for surgery.

During the following years, occasional episodes of headaches, a short period of drowsiness, and difficulty coping with school were reported, but follow-up imaging always showed a normal to narrow ventricle system, and the patient's complaints always ceased a few days later, spontaneously. Multiple ophthalmologic examinations could not identify any abnormalities regarding the fundi, and papillary edema was never reported.

From the age of 6 years, episodes of sudden, severe headaches along with screaming became more and more frequent; however, they resolved just as suddenly, in a few minutes or after some sleep. The patient was hospitalized 3 consecutive times because of the clinical scenarios previously described, but surgery was not indicated, and the symptoms resolved in $24-48$ hours. Retrospectively, from the clinical reports, we know that the opening valve pressure needed multiple readjustments from a low $\left(30 \mathrm{~mm} \mathrm{H}_{2} \mathrm{O}\right.$ ) to a higher level.

At 9 years of age and onward, the patient was hospitalized on multiple occasions for strong (visual analog scale score Io of ro), unbearable headaches accompanied with agitation and screaming. She never presented a progressively reduced level of consciousness or confusion, only moderate, temporary drowsiness. Altogether, we had to revise the shunt system 5 times throughout a year.

Radiologic imaging always showed narrow ventricles. (Figure 1).

Ophthalmologic examinations did not reveal signs of raised ICP.

In 3 of the 5 surgeries, we measured and monitored ICP with an
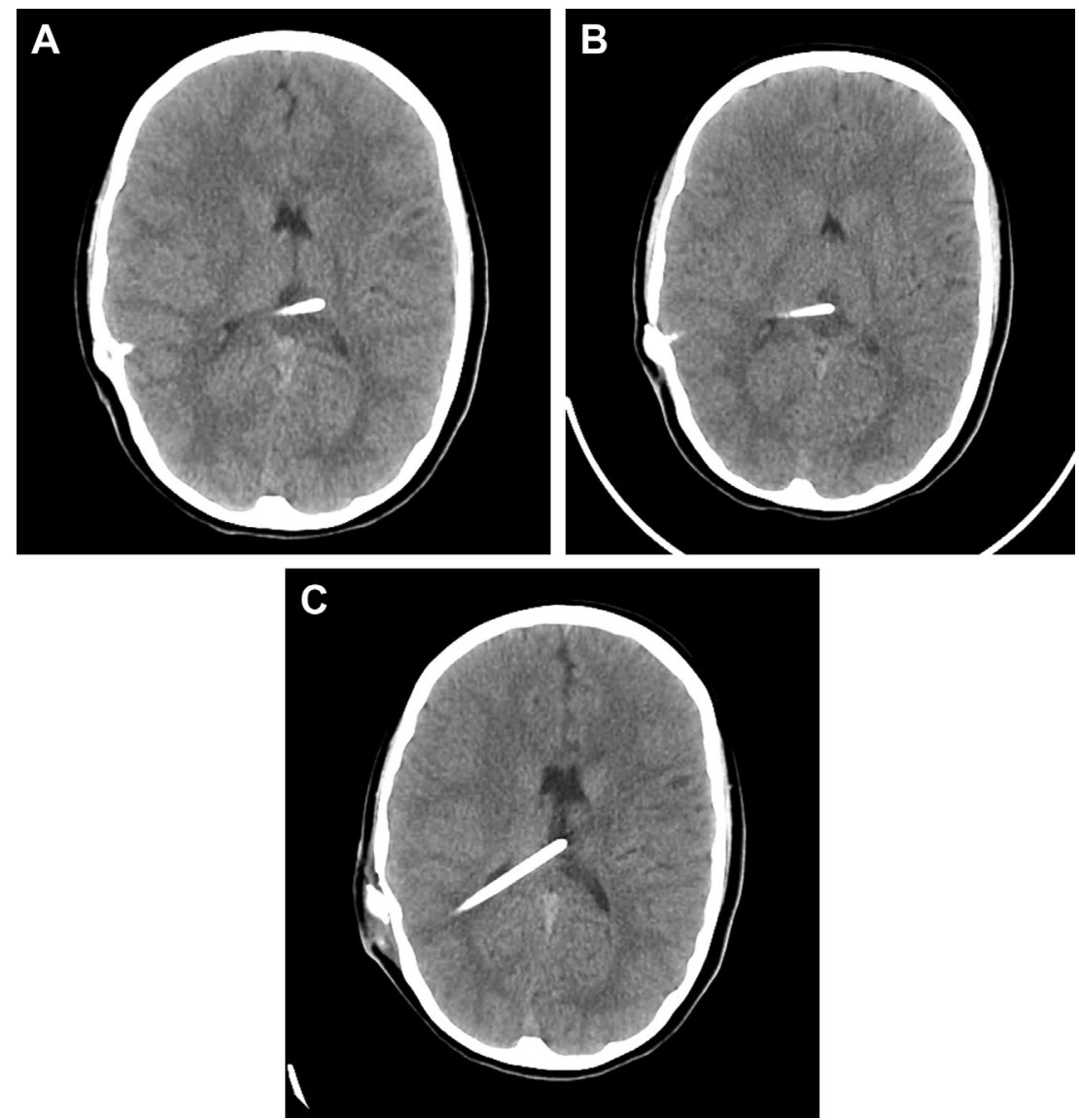

Figure 1. (A-C) Cranial computed tomography (CT) scans. Three axial CT scans taken in the periods of acute strong headaches show no signs of dilated ventricles. The intracranial pressure measured during surgery each time raised above $40 \mathrm{~mm} \mathrm{Hg}$.

intraparenchymal sensor (Pressio [Sophysa, Orsay, France]), which was inserted at the beginning of the operations. Each case gave unexpectedly high values $(40-50 \mathrm{~mm} \mathrm{Hg})$.

During the surgeries, we never found obstruction of any part of the right ventriculoperitoneal shunt system. Cautious movement of the proximal drain resulted in prompt flow of CSF, or a new ventricular drain always restored a satisfactory shunt function for a while. In 2 revisions out of the 5 , the reposition of the ventricular catheter was enough to restore the function of the catheter. In the other 3 cases, once a valve replacement was performed, and twice we changed the ventricular catheters.

A long-term solution could only be achieved by adding another shunt system on the opposite (left) side, with a medium-pressure fixed valve.
In the past 5 years, the patient has experienced no similar symptoms, and follow-up magnetic resonance imaging scans show bilateral narrow ventricles.

\section{DISCUSSION}

In the presented case, the clinical signs and symptoms of raised ICP could not be matched with the radiologic images of narrow/slit ventricles and normal ophthalmologic findings. However, the patient did not show the classical symptoms of raised ICP, whereas the objective values measured with an intraparenchymal sensor were extremely high, and imaging remained insignificant. These findings are contradictory and raise the question of what the rational description of the underlying physiologic events could be.

Regarding the lack of ventricular dilation in case of high-pressure shunt failure, 


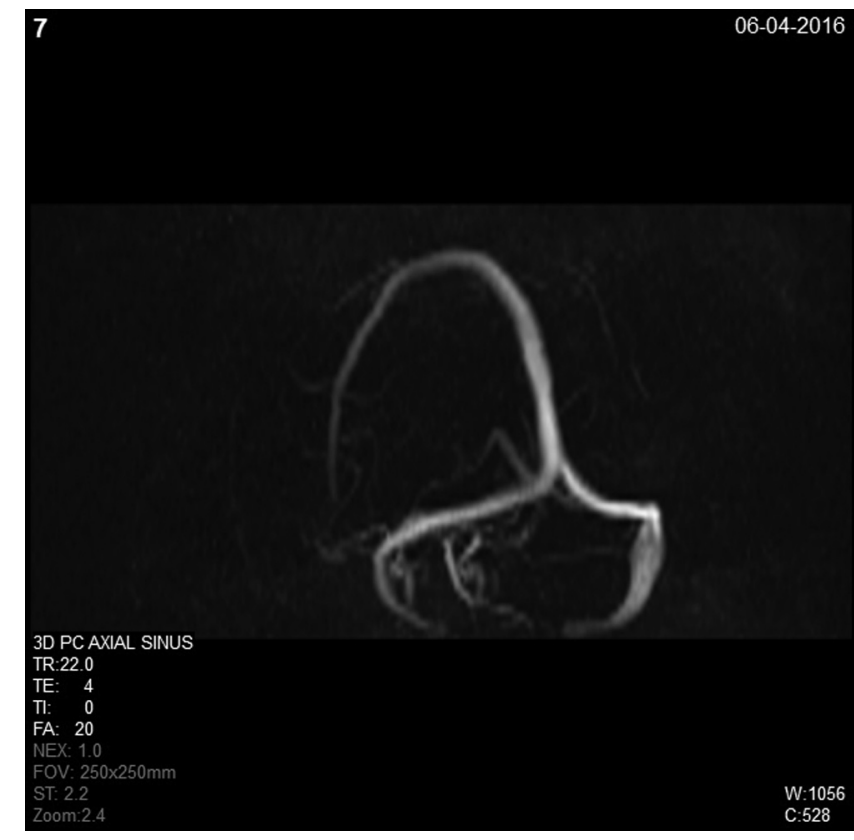

Figure 2. Venous phase magnetic resonance angiography showing patent venous structures of the brain.

the literature puts forward several theories.

\section{Venous Outflow Disturbances}

The brain matter can only change its volume by mobilizing its fluid content. This depends on the venous outflow capacity. Because the venous outflow is limited or blocked, the brain becomes rigid and incompressible; therefore, the ventricular system cannot enlarge. In adults, pseudotumor cerebri is often associated with morphologically proven venous outflow disturbances, ${ }^{2,9-\mathrm{I} 4}$ raised ICP without enlarged ventricles. In the presented case, we could not identify any abnormalities in the venous outflow system: the venous sinuses were intact and had no stenosis or obstruction (Figure 2), but the venous hypertension is the most likely part of the pathomechanism.

\section{Arterial Pulsatility Vector Changes}

The drainage of CSF by a shunt system substantially alters the spread of arterial pulsatility waves onto the CSF space. The arterial pulsatility vector may be abolished with the subsequent loss of the diastolic recurrent wave of the subarachnoid space and its effect at the cortical surface.
Capillary venous outflow of the cortex will be changed because of a lack of diastolic waves. Once the level of ICP reaches the venous pressure of the bridging vessels, they will collapse, and the venous outflow resistance will rise. ${ }^{15}$ The simultaneous increase of venous pressure and ICP in children with hydrocephalus has been reported for many years, ${ }^{\mathrm{I}, \mathrm{I} 6-\mathrm{I} 8}$ especially in those who, as a response to shunt malfunction, present with slit ventricle syndrome. ${ }^{\text {I9 }}$ These findings were interpreted as an early collapse of bridging veins or obstruction of the sinuses. $^{\text {I2,13 }}$

\section{Isolated Ventricle}

Chronic shunting may lead to ventricular asymmetry, and even to the collapse of the ipsilateral ventricle and a functional blockage of the proximal drain. This consequently may cause contralateral ventricular dilation (Figure 3), and if the foramen of Monro becomes closed because of displacement of the septum pellucidum, it may become isolated. ${ }^{3,20,2 \mathrm{I}}$ Typically, this mechanism would cause intermittent, fluctuating clinical symptoms, like in the presented case. The right-side ventricles were always collapsed, whereas the left-side ventricles were moderately dilated. Retrospectively, this mechanism is proven by the longterm benefit of shunt insertion into the left ventricle (where porencephaly was also present).

Theory of Capillary Absorption Laziness This concept argues that chronically altered CSF absorption pathways at the capillary level will ultimately damage normal CSF outflow dynamics, and will lead to high-pressure slit ventricle syndrome. $^{22}$ The normal transcapillary absorption is practically abolished because of an easier outflow offered by shunt insertion. A large part of CSF is drained by the functioning shunt, and the transmural CSF pressure is minimized, which progressively results in the blockage of transcapillary absorption, causing rigidity and incompressibility of brain parenchyma. Our patient's longlasting abnormal physiologic conditions might have contributed to these mechanisms as well.

Our examinations proved that there was no morphologic venous outflow obstruction, but the venous hypertension could persist without abnormal dural venous anatomy, ${ }^{14}$ and all other mechanisms could have played a role in the clinical evolution of this case. However, most probably, the low opening pressure of the valve $\left(30 \mathrm{~mm} \mathrm{H}_{2} \mathrm{O}\right)$ must have caused chronic CSF overdrainage with all its harmful consequences. ${ }^{3,18}$ We cannot fully understand why the valve setting was changed to repeatedly low values.

Furthermore, isolated ventricle syndrome also developed during critical acute periods. The temporary blockage of the foramen of Monro because of the collapse of midline structures made the left-sided ventricle close, the CSF become trapped, and the ventricle become isolated. From this moment, the retrograde flow will be impaired from the subarachnoid spaces to the ventricle and from the left side to the right. This is what we could identify on the follow-up CT scans on occasions of complaints: the left-side ventricle was moderately dilated, whereas the structures on the right side collapsed.

The pressure-volume dynamic features are rather similar to those of patients with severe traumatic brain injuries: a smal 


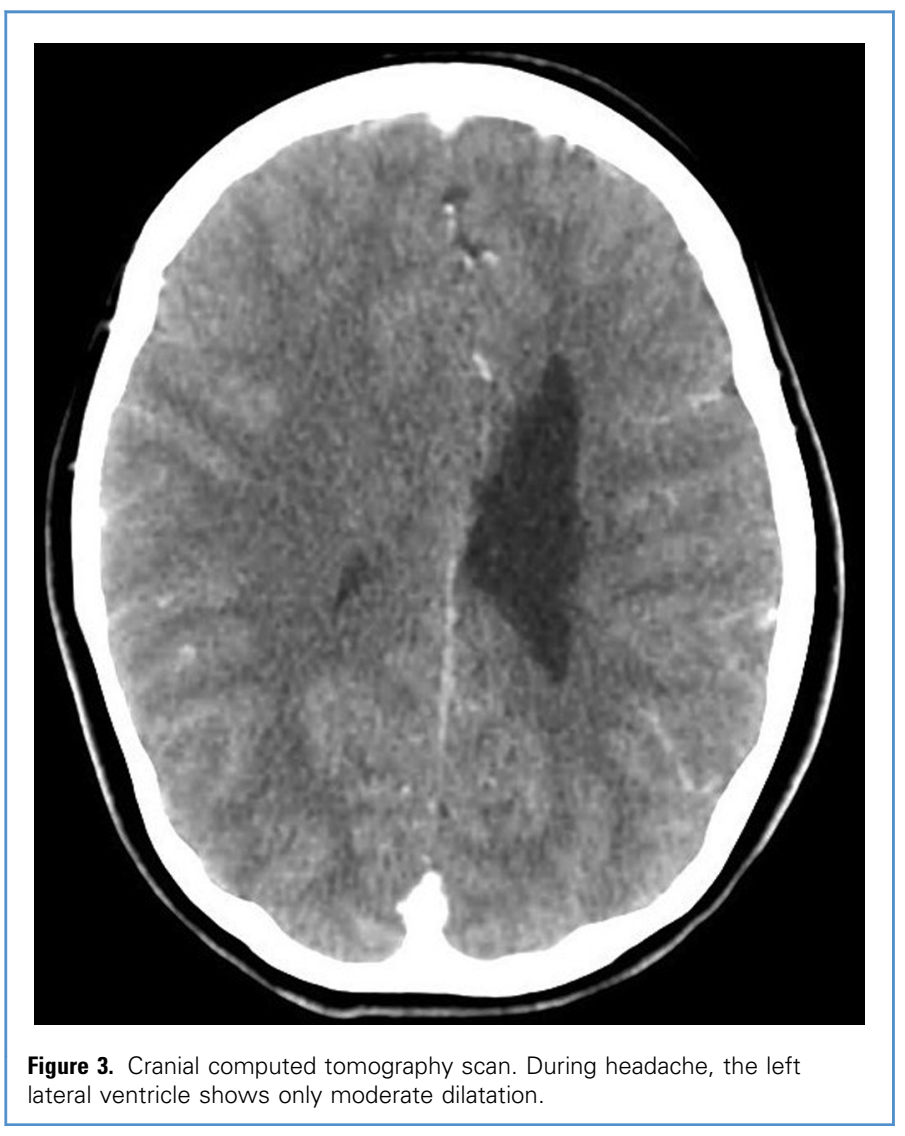

add up (of CSF) to intracranial volume increases the ICP disproportionately. ${ }^{23-25}$

Presumably, children with a shunt system probably have a different capacity of autoregulation, which may ensure appropriate cerebral perfusion even with elevated ICP. ${ }^{17,18,24}$ However, in cases of slit ventricle syndrome, the autoregulation capacity might be exhausted, and minimal increase in CSF volume may provoke a sharp increase in ICP. These pressurevolume reactions are very similar to those experienced in severe traumatic brain injury, depending on the segment of the pressure-volume curve of the patient's vascular compliance. ${ }^{8}$

We think in the presented case that a significant, long-term drainage of CSF (overdrainage) stopped the CSF absorption into the dural venous sinuses by keeping the ICP low and prompted the consequent disturbance in venous circulation. The complex process and the effect of long-term shunt treatment I) caused ventricular asymmetry with recurrent obstructions of the foramen of Monro leading to pressure differences and blocking of the CSF flow, 2) caused insufficient capillary absorption, or 3) induced a collapse of bridging veins with facilitation of an increase in venous hypertension, and eventually the slit ventricle syndrome. . $^{37,18,22}$

The management of symptomatic slit ventricle widely varies from pain management $^{26}$ and repeated shunt revisions ${ }^{27}$ to different extracerebral decompressive surgeries (subtemporal craniectomy and cranial expansion). ${ }^{28,29}$ In the literature, shunt revision generally stands for the change of the ventricle catheter, an insertion of a new valve of higherpressure threshold or antisiphon device. ${ }^{27}$ The literature reports successful decompressive procedures, aiming to reduce the pressure exercised to the brain and the extra-intracerebral CSF pathways. The underlying idea is to keep the ventricles open around the catheter and prevent its isolation. By normalizing the pressure of extracerebral CSF spaces, the collapse of the bridging veins and secondary venous stagnation can also be prevented.

Albright and Tyler-Kabara ${ }^{30}$ performed craniotomy and craniectomy for early ossification of the skull because of shunt overdrainage, which successfully treated the symptoms of slit ventricle. Others found this type of management to be effective only for a limited period of time, which warranted another decompressing intervention, either a combined, ventriculo- and lumboperitoneal shunt ${ }^{31}$ or cisterna magna-peritoneal shunt insertion. Finally, application of a combined cisterna-magna and ventriculoperitoneal shunt could ensure permanent recovery of the patient. ${ }^{32}$

Retrospectively, we think that left-side porencephaly and brain atrophy prevented the lateral ventricle from collapse, which could have an effect similar to extracerebral decompression (lumboperitoneal shunt or craniectomy). Normal CSF outflow could ensure equal pressure conditions between the 2 lateral ventricle systems. The elimination of the difference in pressure (with both sides of shunt placement) could reopen the foramen of Monro and prevent recurrent blockage. The benefit from the insertion of another shunt system into the left ventricular system justifies this idea because the patient has presented no symptoms since then, she only has occasional headaches without neurologic signs. This theory has been confirmed by a dog model: when CSF was withdrawn from one lateral ventricle, dogs showed intraventricular pressure differentials. The foramen of Monro acts as a valve mechanism that usually closes in response to CSF withdrawal.

Since the contralateral ventricles were shunted 5 years ago, the presented patient has been doing well. The beneficial effect of the contralateral shunting may be attributed to the fact that avoidance of ventricular isolation keeps the way to shunts that provide CSF drainage. When a surgeon is developing surgical strategies to treat patients with slit ventricle syndrome, all of these mechanisms should be taken into consideration.

We have to acknowledge that the narrow ventricles, the lack of a significant fall in the level of consciousness, and the lack 
of papillary edema delayed us in recognizing the extremely high ICP.

In fact, in slit ventricle syndrome cases, the real diagnostic challenge is to define high (critical) ICP without ventricular dilation.

As previously discussed, raised ICP can evolve without progressive deterioration in consciousness.

\section{CONCLUSIONS}

We aimed to highlight the importance of ICP measurement ${ }^{33,34}$ to define the pathophysiologic background of shunt malfunction and to give an overview of the literature about possible mechanisms of slit ventricle syndrome, which is a special case of shunt failure.

The presented case, when mild-tomoderate clinical signs and irrelevant radiologic findings did not appropriately reflect the highly abnormal pressure conditions, was rather challenging to manage. Because radiologic images of the slit ventricle can equally be associated with either low or extremely high ICP, the clinical observation and ophthalmologic findings must be completed with ICP. Only its values can indicate adequate surgical management.

Children having had previous shunt insertion and showing a sudden onset of irritability or a decreased level of consciousness might have threateningly high ICP, even without any evidence of ventricular dilation, and might need urgent surgical consideration. Although many medical and surgical treatment modalities are recommended in the literature, the slit ventricle syndrome can effectively be managed also by bilateral shunting of the ventricular system.

\section{REFERENCES}

I. Engel M, Carmel PW, Chutorian AM. Increased intraventricular pressure without ventriculomegaly in children with shunts: "normal volume" hydrocephalus. Neurosurgery. 1979;5:549-552.

2. Bateman GA. Hypertensive slit ventricle syndrome: pseudotumor cerebri with a malfunctioning shunt? J Neurosurg. 2013;119:I503-I5IO.

3. Ros B, Iglesias S, Martín Á, Carrasco A, Ibáñez G, Arráez MA. Shunt overdrainage syndrome: review of the literature. Neurosurg Rev. 2018;4I:969-98I.
4. Rekate HL. Shunt-related headaches: the slit ventricle syndromes. Childs Nerv Syst. 2008;24: $423-430$.

5. Rekate HL. Classification of slit-ventricle syndromes using intracranial pressure monitoring. Pediatric Neurosurg. I993;I9:I5-20.

6. Chernov MF, Kamikawa S, Yamane F, Ishihara S, Hori T. Neurofiberscope-guided management of slit-ventricle syndrome due to shunt placement. J Neurosurg. 2005;102(3 suppl):260-267.

7. Eide PK. Quantitative analysis of continuous intracranial pressure recordings in symptomatic patients with extracranial shunts. J Neurol Neurosurg Psychiatry. 2003;74:23I-237.

8. Marmarou A, Shulman K, Lamorgese J. Compartmental analysis of compliance and outflow resistance of the cerebrospinal fluid system. J Neurosurg. I975;43:523-534.

9. Pickard JD, Czosnyka Z, Czosnyka M, Owler B, Higgins JN. Coupling of sagittal sinus pressure and cerebrospinal fluid pressure in idiopathic intracranial hypertension - a preliminary report. Acta Neurochir Suppl. 2008;102:283-285.

Io. Dwyer CM, Prelog K, Owler BK. The role of venous sinus outflow obstruction in pediatric idiopathic intracranial hypertension. J Neurosurg Pediatr. 20I3;II:I44-I49.

II. Sainte-Rose CS, Lacombe J, Pierre-Kahn A, Renies D, Hirsch J-F. Intracranial venous sinus hypertension: cause or consequence of hydrocephalus in infants? J Neurosurg. I984;60:727-736.

I2. Bateman GA, Lechner-Scott J, Copping $\mathrm{R}$, Moeskops C, Yap SL. Comparison of the sagittal sinus cross-sectional area between patients with multiple sclerosis, hydrocephalus, intracranial hypertension and spontaneous intracranial hypotension: a surrogate marker of venous transmural pressure? Fluids Barriers CNS. 2017;14:I8.

I3. Bateman GA, Smith RL, Siddique SH. Idiopathic hydrocephalus in children and idiopathic intracranial hypertension in adults: two manifestations of the same pathophysiological process? J Neurosurg. 2007;107(6 suppl):439-444.

I4. Karahalios DG, Rekate HL, Khayata MH Apostolides PJ. Elevated intracranial venous pressure as a universal mechanism in pseudotumo cerebri of varying etiologies. Neurology. 1996;46: Ig8-202.

15. Preuss M, Hoffmann KT, Reiss-Zimmermann M, et al. Updated physiology and pathophysiology of CSF circulation - the pulsatile vector theory. Childs Nerv Syst. 2013;29:I8II-I825.

I6. Fried A, Shapiro K. Subtle deterioration in shun ted childhood hydrocephalus. A biomechanical and clinical profile. J Neurosurg. I986;65:2II-2I6.

I7. Shapiro K, Fried A. Pressure-volume relationships in shunt-dependent childhood hydrocephalus. The zone of pressure instability in children with acute deterioration. J Neurosurg. I986;64:390-396.
I8. Di Rocco C, Massimi L, Tamburrini G. Shunts vs endoscopic third ventriculostomy in infants: are there different types and/or rates of complications? A review. Childs Nerv Syst. 2006;22:1573-I589.

I9. Shapiro K, Fried A, Marmarou A. Biomechanical and hydrodynamic characterization of the hydrocephalic infant. J Neurosurg. I985;63:69-75.

20. Linder M, Diehl JT, Sklar FH. Significance of postshunt ventricular asymmetries. J Neurosurg. I98I; 55:I83-I86.

2I. Rekate HL, Williams FC Jr, Brodkey JA, McCormick JM, Chizeck HJ, Ko W. Resistance of the foramen of Monro. Pediatr Neurosci. I988; I4: 85-89.

22. Jang M, Yoon SH. Hypothesis for intracranial hypertension in slit ventricle syndrome: new concept of capillary absorption laziness in the hydrocephalic patients with long-term shunts. Med Hypotheses. 20I3;8I:I99-20I.

23. Haubrich C, Czosnyka M, Diehl R, Smielewski P, Czosnyka Z. Ventricular volume load reveals the mechanoelastic impact of communicating hydrocephalus on dynamic cerebral autoregulation. PLoS One. 2016;II:eor58506.

24. Adams H, Donnelly J, Czosnyka M, et al. Temporal profile of intracranial pressure and cerebrovascular reactivity in severe traumatic brain injury and association with fatal outcome: an observational study. PLoS Med. 2017;I4:eI002353.

25. Szczepański TA, Weiser A, Zub WL, Jarmundowicz W, Koźba-Gosztyła M, Czapiga B. Assessment of cerebral blood flow during infusion test in the diagnosis of normal pressure hydrocephalus. Adv Clin Exp Med. 2012;21:55-6I.

26. Obana WG, Raskin NH, Cogen PH, Szymanski JA Edwards MS. Antimigraine treatment for slit ventricle syndrome. Neurosurgery. I990;27:760-763 [discussion: 763 ].

27. Czosnyka M, Czosnyka Z. Overdrainage of cerebrospinal fluid and hydrocephalus shunts. Acta Neurochir (Wien). 2017;159:1387-I388.

28. Epstein FJ, Fleischer AN, Hochwald GM Ransohoff J. Subtemporal craniectomy for recurrent shunt obstruction secondary to small ventricles. J Neurosurg. I974;4I:29-3I.

29. Buxton N, Punt J. Subtemporal decompression: the treatment of noncompliant ventricle syndrome. Neurosurgery. I999;44:513-518 [discussion: 518-519].

30. Albright AL, Tyler-Kabara E. Slit-ventricle syndrome secondary to shunt-induced suture ossification. Neurosurgery. 200I;48:764-770.

31. Le H, Yamini B, Frim DM. Lumboperitoneal shunting as a treatment for slit ventricle syndrome. Pediatr Neurosurg. 2002;36:178-182.

32. Rekate HL, Nadkari T, Wallace D. Severe intracranial hypertension in slit ventricle syndrome managed using a cisterna magna-ventricle-peritoneum shunt. J Neurosurg. 2006; I04(4 suppl): 240-244. 
33. Sæhle $\mathrm{T}$, Eide PK. Intracranial pressure monitoring in pediatric and adult patients with hydrocephalus and tentative shunt failure: a singlecenter experience over Io years in 146 patients. J Neurosurg. 2015;122:1076-1086.

34. Eide PK, Sorteberg W. Diagnostic intracranial pressure monitoring and surgical management in idiopathic normal pressure hydrocephalus: a 6- year review of 2I4 patients. Neurosurgery. 2010;66: 8o-9I.

Conflict of interest statement: The authors declare that the article content was composed in the absence of any commercial or financial relationships that could be construed as a potential conflict of interest.

Received 14 April 2019; accepted 1 July 2019
Citation: World Neurosurg. (2019) 130:493-498. https://doi.org/10.1016/j.wneu.2019.07.006

Journal homepage: www.journals.elsevier.com/worldneurosurgery

Available online: www.sciencedirect.com

1878-8750/\$ - see front matter (C) 2019 Elsevier Inc. All rights reserved. 